\title{
Cuidados Paliativos: Por que Precisamos Falar sobre isso?
}

doi: https://doi.org/10.32635/2176-9745.RBC.2019v65n4.558

\author{
Palliative Care: Why do we Need to Talk about it? \\ Cuidados Paliativos: ¿Por qué Tenemos que Hablar al Respecto?
}

Livia Costa de Oliveira ${ }^{1}$

\section{INTRODUÇÃO}

A expressiva prevalência das doenças crônicas não transmissíveis, resultante da transição epidemiológica e demográfica, é a causa principal da incapacidade de uma parcela importante da população mundial ${ }^{1}$. A expectativa para 2030 é de que haja mais de um bilhão de idosos no mundo, com aumento mais acentuado desse grupo etário nos países em desenvolvimento. Por conseguinte, as doenças crônicas não transmissíveis representarão mais de 3/4 de todas as mortes, com destaque para as doenças cardiovasculares e oncológicas, aumentando a demanda por Cuidados Paliativos ${ }^{2,3}$.

No entanto, essa demanda não é suficientemente suprida e inúmeras barreiras (política; de disponibilidade e acesso a medicamentos opioides; de implementação de serviços que ofereçam esse tipo cuidado; e a necessidade de educar a sociedade, técnica e leiga, a respeito dessa temática) precisam ser superadas, corroborando que, de fato, os Cuidados Paliativos possam ser prestados aos indivíduos como um direito humano legítimo, de acordo com os princípios da Cobertura Universal da Saúde ${ }^{4}$.

\section{CUIDADOS PALIATIVOS - DEFINIÇÃO E PRESSUPOSTOS}

A palavra "paliativo" tem origem latina, "pallium", que significa manto, representando o alívio do sofrimento enquanto a condição subjacente/doença não pode ser curada. Trata-se de um tratamento ativo que envolve o indivíduo e tudo o que é inerente a ele em cuidados perceptíveis; ou seja, tratando-o em sua integralidade e propiciando o empoderamento sobre a sua própria vida e finitude ${ }^{5}$. Cada paciente é composto por um contexto socioeconômico, espiritual e psicológico singular. Esses elementos não são dissociáveis, motivo pelo qual não devem ser considerados dessa forma.
Cuidado Paliativo é definido pela World Health Organization ${ }^{2}$ como uma abordagem que objetiva a melhora da qualidade de vida dos pacientes e de seus familiares diante de uma doença que ameaça a continuidade da vida, por meio da prevenção e alívio do sofrimento, identificação precoce, avaliação e tratamento da dor e de outros sintomas de ordem física, psicossocial e espiritual. Essa definição pode ser estendida, abrangendo indivíduos com condiçôes que exijam Cuidados Paliativos no futuro e aqueles com idade mais avançada que estejam chegando ao fim do curso da vida.

Tem como pressupostos: aliviar sintomas; apoiar os pacientes a viverem o mais ativamente possível; integrar aspectos físicos, psicológicos, espirituais e sociais; afirmar a vida e a morte como processos naturais; e apoiar amigos e familiares a lidarem com o paciente e com o luto diante da finitude da vida ${ }^{2}$. Esse tipo de cuidado deve ser iniciado no surgimento de quaisquer manifestaçôes de uma condição/doença ameaçadora da vida, em conjunto com as terapêuticas capazes de modificar seu curso. A paliação ganha maior importância à medida que as terapêuticas curativas perdem sua efetividade ${ }^{6}$.

Os Cuidados Paliativos precoces não apenas melhoram a qualidade de vida dos pacientes e promovem um cuidado digno, mas também reduzem as hospitalizaçôes desnecessárias e o uso de serviços de saúde, contribuindo para a utilização eficiente de recursos ${ }^{2}$. Em relação às doenças oncológicas, a concepção de tratamento abrangente deve contemplar os Cuidados Paliativos ${ }^{7}$. Um crescente corpo de evidências sugere que o envolvimento precoce de Cuidados Paliativos especializados, concomitantemente com os cuidados oncológicos curativos, esteja associado, entre outros fatores, a uma melhor tolerância dos pacientes aos tratamentos mais agressivos, menor carga de sintomas e maior qualidade de vida ${ }^{8-10}$. 
CENÁRIO ATUAL - DEMANDA E ACESSO AOS CUIDADOS PALIATIVOS

A cada ano, mais 40 milhóes de pessoas (incluindo familiares/cuidadores) demandam por Cuidados Paliativos no mundo. Entretanto, apenas $14 \%$ dessas o recebem de fato ${ }^{6}$.

Conforme dados publicados no Global Atlas of Palliative Care at the End of Life ${ }^{6}$, alguns países da América do Norte, Europa e Austrália possuem um bom nível de desenvolvimento de Cuidados Paliativos. Em contraponto, há localidades onde, sequer, há alguma atividade de paliação registrada (por exemplo: Afeganistão, Líbia, Guiana Francesa e Yemen). O Brasil, por sua vez, foi classificado no nível 3A; ou seja, possui apenas alguns serviços desse tipo dispostos em ilhas isoladas no território nacional, correspondendo a apenas 172 equipes registradas e menos de $1 \%$ do número total de hospitais do país.

O The 2015 Quality of Death Index ${ }^{11}$ classificou o Brasil em $42^{\circ}$ lugar em um ranking envolvendo um total de 80 diferentes países, refletindo a necessidade de construção de novos serviços, aprimoramento e difusão do conhecimento em Cuidados Paliativos entre os profissionais de saúde e a sociedade. Além disso, verificou-se que somente $0,3 \%$ das pessoas que morrem anualmente no Brasil tem a possibilidade de receber esse tipo de cuidado.

Em razáo do impacto das doenças oncológicas na populaçáo, quase todas as famílias são afetadas de maneira direta ou indireta. Ademais, nos países em desenvolvimento, as limitaçôes no acesso à saúde, baixa cobertura de programas de rastreamento e atrasos na realizaçáo de procedimentos diagnósticos contribuem para o diagnóstico tardio, e o câncer costuma ser reconhecido já em estágio avançado, cujo prognóstico tende a ser ruim ${ }^{12}$.

Dados do Instituto Nacional de Câncer José Alencar Gomes da Silva (INCA) evidenciam a amplitude dos casos de câncer em fase avançada no Brasil. Dos pacientes matriculados na Unidade de Cuidados Paliativos, 78\% já ingressam no INCA sem critérios de elegibilidade para um tratamento com proposta curativa. Além disso, a taxa de óbito é elevada mesmo em outras unidades de tratamento da instituiçấo, apontando que uma parcela expressiva de pacientes já inicia o tratamento sem condiçóes de cura do câncer e nem chega a ser encaminhada para a Unidade de Cuidados Paliativos exclusivos ${ }^{13}$.

No cenário hospitalar, é comum que pacientes com câncer avançado e sem perspectiva de tratamento modificador da doença recebam assistência inadequada e ineficaz, sem previsão de cuidados paliativos e alívio da dor. Mesmo em uma realidade de recursos escassos, percebe-se a utilização desnecessária de métodos invasivos e de alta tecnologia, focados na tentativa de cura, que se mostram incapazes de tratar os sintomas mais prevalentes da doença, prolongando o sofrimento e a dor ${ }^{14}$. Uma cultura paliativa poderia lentamente contribuir para reversão dessa realidade.

\section{CONCLUSÃO}

Cuidado Paliativo é uma necessidade de saúde pública que, em virtude de uma oferta ainda incipiente, é acessado por uma pequena parcela dos indivíduos que dele necessitam. Há um vasto caminho a ser percorrido rumo à oferta universal e à melhoria desse tipo de cuidado.

Precisamos falar sobre esse tema e colocá-lo em evidência por meio de um sistema de educaçáo/conscientizaçáo para toda a sociedade, gerando novas organizaçóes de consciência que corroborem o desenvolvimento do Cuidado Paliativo, bem como o acesso a ele.

\section{DECLARAÇÃO DE CONFLITO DE INTERESSES}

\author{
Nada a declarar.
}

\section{FONTES DE FINANCIAMENTO}

Não há.

\section{REFERÊNCICAS}

1. Araújo Neto LA. Muito além da transição epidemiológica: doenças crônicas no século XX. Hist Cienc SaúdeManguinhos. 2019;26(1):353-5. doi: http://dx.doi. org/10.1590/s0104-59702019000100022

2. World Health Organization. Palliative care [Internet]. Genebra: WHO; 2018 Feb.19. [cited 2019 Aug 10]. Available from: https://www.who.int/en/news-room/ fact-sheets/detail/palliative-care

3. World Health Organization. Global action plan for the prevention and control of noncommunicable diseases 2013-2020. Geneva: WHO; 2013.

4. Brennen F. Palliative care as an international human right. J Pain Symptom Manage. 2007;33(5):494-9. doi: http:// dx.doi.org/10.1016/j.jpainsymman.2007.02.022

5. Merino MTGB. Palliative care: taking the long view. Front Pharmacol. 2018;9:1140. doi: https://doi. org/10.3389/fphar.2018.01140

6. Connor SR, Bermedo MCS, editors. Global atlas of palliative care at the end of life [Internet]. Geneva: World Palliative Care Alliance; c2014. [cited 2019 Aug 10]. Available from: http://www.who.int/cancer/publications/ palliative-care-atlas/en

7. Bolt EE, Pasman HR, Willems D, et al. Appropriate and inappropriate care in the last phase of life: an explorative study among patients and relatives. BMC Health Serv Res. 2016;16(1):655. doi: https://doi.org/10.1186/ s12913-016-1879-3 
8. Zimmermann C, Swami N, Krzyzanowska M, et al. Early palliative care for patients with advanced cancer: a cluster-randomised controlled trial. Lancet. 2014;383(9930):1721-30. doi: https://doi.org/10.1016/ S0140-6736(13)62416-2

9. Kelley AS, Morrison RS. Palliative care for the seriously Ill. N Engl J Med. 2015;373(8):747-55. doi: https://doi. org/10.1056/NEJMra1404684

10. Hui D, Bruera E. Integrating palliative care into the trajectory of cancer care. Nat Rev Clin Oncol. 2016;13(3):159-71. doi: https://doi.org/10.1038/ nrclinonc.2015.201

11. Economist Intelligence Unit. The 2015 quality of death index: ranking palliative care across the world [Internet]. London: Economist Intelligence Unit; 2015. [cited 2019 Aug 10]. Available from: https://www.eiuperspectives. economist.com/sites/default/files/2015\%20EIU\%20 Quality\%20of\%20Death\%20Index\%20Oct\%2029\%20 FINAL.pdf

12. World Health Organization. Cancer [Internet]. Genebra: WHO; 2016. [cited 2019 Sept 18]. Available from: http:// http://www.who.int/cancer/en/.

13. Instituto Nacional de Câncer José Alencar Gomes da Silva. Relatório de gestão INCA 2018. Rio de Janeiro: INCA; 2018.

14. Carvalho RCT, Parsons HA, organizadores. Manual de cuidados paliativos. 2. ed. ampl. atual. São Paulo: Academia Nacional de Cuidados Paliativos; 2012. 